\title{
Voronoi Diagrams and Convex Hulls of Random Moving Points
}

\author{
R. A. Dwyer \\ Department of Computer Science, North Carolina State University, \\ Raleigh, NC 27695-8206, USA \\ dwyer@csc.ncsu.edu
}

\begin{abstract}
This report considers the expected combinatorial complexity of the Euclidean Voronoi diagram and the convex hull of sets of $n$ independent random points moving in unit time between two positions drawn independently from the same distribution in $\mathrm{R}^{d}$ for fixed $d \geq 2$ as $n \rightarrow \infty$. It is proved that, when the source and destination distributions are the uniform distribution on the unit $d$-ball, these complexities are $\Theta\left(n^{(d+1) / d}\right)$ for the Voronoi diagram and $O\left(n^{(d-1) /(d+1)} \log n\right)$ for the convex hull. Additional results for the convex hull are $O\left(\log ^{d} n\right)$ for the uniform distribution in the unit $d$-cube and $O\left(\log ^{(d+1) / 2} n\right)$ for the $d$-dimensional normal distribution.
\end{abstract}

\section{Introduction}

Convex hulls and Voronoi diagrams of various ilks have been staples of computational geometry since its debut as an identifiable field with the publication of Shamos's dissertation [14]. While attention has more frequently been focused on the worst-case combinatorial complexity of these structures, some investigators have also considered average complexities for various input distributions [1], [6], [8], [10], [11].

Recently, Guibas et al. [9], Albers and Roos [3], and Agarwal et al. [2] have considered the worst-case complexity for sets of moving points in $\mathrm{R}^{d}$. The strongest of these results shows that the number of distinct topologies assumed by the Voronoi diagram is $O\left(n^{d+1}\right)$ $(d \geq 3)$ and by the convex hull, $O\left(n^{d}\right)$ and $\Omega\left(n^{\lfloor d / 2\rfloor+1}\right)$.

This work comprises asymptotic analyses of the average combinatorial complexity of the Voronoi diagram and convex hull for sets of independent random moving points in $\mathrm{R}^{d}$. We define a random moving point to be one moving with unvarying velocity between a random source and an independent but identically distributed (i.i.d.) random destination during the time interval $[0,1]$. Our results may be regarded as generalizations of recent results for the plane conjectured by Roos [12] and subsequently derived by Basch et al. [4] 
(although the distributions differ somewhat). The following three theorems summarize our work:

Theorem 1. For any fixed $d \geq 2$, the expected combinatorial complexity of the Euclidean Voronoi diagram of $n$ random points moving between source and destination chosen from the uniform distribution on the unit d-ball is $\Theta\left(n^{(d+1) / d}\right)$ as $n \rightarrow \infty$.

Theorem 2. Let $\mathcal{B}_{j}$ denote the unit ball in $j$ dimensions, and consider a fixed $d$ dimensional convex body

$$
\mathcal{K}=\mathcal{B}_{d_{1}} \times \mathcal{B}_{d_{2}} \times \cdots \times \mathcal{B}_{d_{k}}
$$

where $d_{1}=d_{2}=\cdots=d_{m}>d_{m+1} \geq d_{m+2} \geq \cdots \geq d_{k}$ and $\sum_{i=1}^{k} d_{i}=d$. The expected combinatorial complexity of the convex hull of $n$ random points moving between source and destination chosen from the uniform distribution on $\mathcal{K}$ is $O\left(n^{\left(d_{1}-1\right) /\left(d_{1}+1\right)} \log ^{m} n\right)$ as $n \rightarrow \infty$.

Theorem 3. For any fixed $d \geq 2$, the expected combinatorial complexity of the convex hull of $n$ random points moving between source and destination chosen independently from the standard normal distribution in $\mathrm{R}^{d}$ is $\Theta\left(\log ^{(d+1) / 2} n\right)$ as $n \rightarrow \infty$.

The conditions of Theorem 2 deserve some comment. If $k=m=1$ and $d_{1}=d$, $\mathcal{K}$ is just a $d$-ball and the result is $O\left(n^{(d-1) /(d+1)} \log n\right)$. If $k=m=d$ and $d_{i}=1$ for all $i: 1 \leq i \leq k$, then $\mathcal{K}$ is a $d$-cube and the bound is $O\left(\log ^{d} n\right)$. This family of distributions has been investigated previously in the context of convex hulls of static point sites [5].

Although the particular distributions analyzed here may be unlikely in practice, they are almost certainly more indicative of "typical" complexity than the worst-case bounds. It is reasonable to conjecture, for example, that our near-linear bounds hold whenever the endpoints of the line segments are chosen from any absolutely continuous distribution. Therefore, this result should be encouraging to those who seek to construct such diagrams for practical applications.

For every distribution covered by either Theorem 2 or Theorem 3, movement adds a factor of $\Theta(\log n)$ to the bounds known for static points. A proof of a general theorem, however, seemed elusive. A characterization of the distributions for which movement adds no more than $O(\log n)$ to the combinatorial complexity of the convex hull remains as an interesting open problem.

The remainder of this paper is organized as follows: In the next section we introduce notation and formalize our notion of "random moving point." Section 3 derives a density transformation that allows us to express a set of $d+2$ moving points in terms of a common hypersphere, a form particularly convenient for analyzing Voronoi diagrams. In Section 4 this transformation is applied to complete the proof of Theorem 1. Sections 5 and 6 derive a density transformation for convex hulls and complete the proof of Theorem 2 . In Section 7 we sketch the proof of Theorem 3. 


\section{Definitions and Preliminaries}

Let $\mathcal{P}_{n}=\left\{P_{1}, P_{2}, \ldots, P_{n}\right\}$ be a set of moving points in $\mathrm{R}^{d}$ for some fixed $d \geq 2$. By "moving point" we mean a continuous function $P:[0,1] \rightarrow \mathrm{R}^{d}$. At any time $t \in[0,1]$, we can compute the Voronoi diagram of the set $\mathcal{P}_{n}(t)=\left\{P_{1}(t), P_{2}(t), \ldots, P_{n}(t)\right\}$, and, the points themselves being in continuous motion, we find that the Voronoi diagram, too, evolves continuously.

However, the topological structure of the Voronoi diagram is constant over certain finite time intervals. In particular, if $\delta(\cdot, \cdot)$ denotes Euclidean distance, the edge set of the dual graph (Delauney triangulation)

$$
\begin{aligned}
D G\left(\mathcal{P}_{n}(t)\right)= & \left\{(i, j) \mid \exists\left(x \in \mathrm{R}^{d}\right) \forall(k \in\{1, \ldots, n\}) \delta\left(P_{i}(t), x\right)=\delta\left(P_{j}(t), x\right)\right. \\
& \left.\leq \delta\left(P_{k}(t), x\right)\right\}
\end{aligned}
$$

changes only at discrete instants in time. In fact, Albers and Roos [3] observed that these transitions occur exactly when $d+2$ points lie on a common "empty" sphere (containing none of the other $n-d-2$ points), and that this implies a $O\left(n^{d+2}\right)$ bound (not tight) on the number of such transitions.

Since the worst-case and average complexities of the usual Voronoi diagram of stationary points are vastly different- $\Theta\left(n^{\lfloor(d+1) / 2\rfloor}\right)$ and $\Theta(n)$, respectively-it is of interest to know whether the $\Theta\left(n^{d+2}\right)$ bound is "typical." To provide a relevant answer to this question, we must devise a probability distribution of moving points that is "natural," i.e, not contrived to simplify analysis, but that nonetheless renders analysis feasible. To meet these criteria, we define

$$
P_{i}(t):=(1-t) X_{i}+t Y_{i},
$$

where $X_{i}$ and $Y_{i}$ are chosen independently from the uniform distribution on the unit ball. Thus, a "random moving point" is one that travels in a straight line at an unvarying speed between two points chosen at random from the unit ball.

The usual Lebesgue measure on $\mathrm{R}^{d}$ ( $d$-volume) is denoted by $\lambda_{d}$. Slightly abusively, we use $B_{d}$ to denote both the unit $d$-ball and its volume, so that

$$
B_{d}=\lambda\left(B_{d}\right)=\frac{2 \pi^{d / 2}}{d \Gamma(d / 2)} .
$$

We use $\sigma_{k-1}$ to denote the usual rotation-invariant measure of orientations in $\mathrm{R}^{k}$. This may also be viewed as a measure on the space of (unit) vectors on the $(k-1)$-dimensional unit sphere (the boundary of the unit $k$-ball). $S_{d-1}$ denotes both the unit $(d-1)$-sphere and its $(d-1)$-volume ("surface area"), thus

$$
S_{d-1}=\int_{S_{d-1}} d \sigma_{d-1}(u)=d \cdot B_{d}=\frac{2 \pi^{d / 2}}{\Gamma(d / 2)} .
$$

In order to analyze the expected number of Voronoi transitions, it will be necessary to express the $d+2$ moving points defining the transition in terms of the transition itself rather than in terms of the origin of the standard coordinate system. In the next section 
we describe such a system of coordinates for the lines and derive the corresponding density transformation. The density transformation could be derived by considering the $\left(d^{2}+2 d\right) \times\left(d^{2}+2 d\right)$ Jacobian determinant of the coordinate transformation, as done elsewhere for $d+1$ stationary points [6]. Instead, we use the more concise notations and techniques of the exterior algebra of differential forms, as presented by Santaló [13, Chapter 12], to carry out this task. This notation centers around the anticommutative wedge product, which satisfies

$$
d x \wedge d y=-d y \wedge d x \text { and (therefore) } \quad d x \wedge d x=0 .
$$

To illustrate briefly, we rederive the well-known density transformation $d x d y=r d r d \theta$ relating Cartesian and polar coordinates in the plane. Since $x=r \cos \theta$ and $y=r \sin \theta$, we have

$$
\begin{aligned}
d x \wedge d y= & (-r \sin \theta d \theta+\cos \theta d r) \wedge(r \cos \theta d \theta+\sin \theta d r) \\
= & (-r \sin \theta d \theta) \wedge(r \cos \theta d \theta)+(-r \sin \theta d \theta) \wedge(\sin \theta d r) \\
& +(\cos \theta d r) \wedge(r \cos \theta d \theta)+(\cos \theta d r) \wedge(\sin \theta d r) \\
= & 0+\left(r \sin ^{2} \theta d r \wedge d \theta\right)+\left(r \cos ^{2} \theta d r \wedge d \theta\right)+0 \\
= & r d r \wedge d \theta .
\end{aligned}
$$

In higher dimensions, this formula becomes

$$
\bigwedge_{j=1}^{d} d x^{(j)}=d \lambda(x)=|x|^{d-1} d|x| d \sigma_{d-1}(x /|x|) .
$$

Two related identities—for unit vectors $u=\left(u^{(1)}, u^{(2)}, \ldots, u^{(d)}\right)$ only-are

$$
d \sigma_{d-1}(u)=\frac{1}{u^{(1)}} \bigwedge_{i=2}^{d} d u^{(i)} \quad \text { and } \quad d \sigma_{d-1}(u)=\sin ^{d-2} \psi d \psi d \sigma_{d-2}\left(u^{\prime}\right),
$$

where $\psi$ is the angle between $u$ and the positive $x^{(d)}$-axis, and $u^{\prime}$ is a unit vector in the hyperplane $x^{(d)}=0$.

For the inner product of two vectors $x_{1}, x_{2} \in \mathrm{R}^{d}$, we use the notation $\left\langle x_{1}, x_{2}\right\rangle:=$ $\sum_{j=1}^{d} x_{1}^{(j)} x_{2}^{(j)}$. Simple but useful consequences of the multiplication rule $d(u v)=u d v+$ $v d u$ are [13]

$$
\begin{aligned}
& d\left\langle x_{1}, x_{2}\right\rangle=\left\langle d x_{1}, x_{2}\right\rangle+\left\langle x_{1}, d x_{2}\right\rangle, \\
& \left\langle d x_{1}, x_{2}\right\rangle=-\left\langle x_{1}, d x_{2}\right\rangle \quad \text { if } \quad x_{1} \perp x_{2}, \\
& \left\langle d x_{1}, x_{1}\right\rangle=\frac{1}{2} d\left\langle x_{1}, x_{1}\right\rangle=\frac{1}{2} d\left(\left|x_{1}\right|^{2}\right)=\left|x_{1}\right| d\left|x_{1}\right|,
\end{aligned}
$$

where $d x_{i}$ is the vector $\left(d x_{i}^{(1)}, d x_{i}^{(2)}, \ldots, d x_{i}^{(d)}\right)$.

The following geometric lemma, proved in detail in an earlier paper [7, p. 268], will be used repeatedly:

Lemma 1. Let $L_{d}(q, r)$ represent the volume of the lens formed by intersecting the unit $d$-ball with a ball of radius $r$ centered at distance $q$ from the origin. If $r \geq 1$, then $L_{d}(q, r)=O\left(\min \left(1,(1-q+r)^{(d+1) / 2}\right)\right)$. 
Now we turn our attention to the distribution of points at time $t$.

Lemma 2. Let $f_{t}(p)$ be the density function of the probability that a random moving point is at point $p \in \mathrm{R}^{d}$ at fixed time $t \leq \frac{1}{2}$. Then

$$
f_{t}(P)=(1-t)^{-d} L_{d}(|P| / t,(1-t) / t)
$$

Proof. Let $P=P(x, y)$ be a random moving point. At time $t$, its position is $P=$ $(1-t) x+t y$. Thus, $x=(P-t y)(1-t)^{-1}$ and

$$
\begin{aligned}
d x^{(j)} & =\left(P^{(j)}-t y(j)\right)(1-t)^{-2} d t+\left(d P^{(j)}-t d y^{(j)}+y^{(j)} d t\right)(1-t)^{-1} \\
& =(1-t)^{-1}\left(d P^{(j)}-t d y^{(j)}+\left(x^{(j)}-y^{(j)}\right) d t\right) \\
d x^{(j)} \wedge d y^{(j)} & =(1-t)^{-1}\left(d P^{(j)} \wedge d y^{(j)}\right)
\end{aligned}
$$

since $t$ is fixed. So

$$
\left.d \lambda(x) \wedge d \lambda(y)\right|_{\text {fixed } t}=(1-t)^{-d} d \lambda(y) \wedge d \lambda(P),
$$

and the density $f_{t}(P)$ is proportional to the Lebesgue measure of the set $\mathcal{Y}$ of possible eventual destinations of the point (within the unit $d$-ball).

Now let $\mu(X)$ be the destination point of a moving point with source $X$ passing through $P$ at time $t$. Then

$$
\mu(X)=X+(P-X) / t=P / t-X(1-t) / t
$$

Now $\mathcal{Y}$ is a ball with center $\mu(O)=P / t$ and radius $|\mu((1,0,0, \ldots, 0))-\mu(O)|=$ $(1-t) / t$. Thus, $f_{t}(P)=(1-t)^{-d} \lambda(\mathcal{Y})=(1-t)^{-d} L_{d}(|P| / t,(1-t) / t)$.

\section{A Density Transformation for Voronoi Diagrams}

This section describes a convenient system of coordinates for expressing the $d+2$ moving points defining a Voronoi transition and derives the density transformation corresponding to these coordinates.

Let $P_{i}=P_{i}\left(x_{i}, y_{i}\right)$ for $1 \leq i \leq d+2$ be the initial and final positions of $d+2$ moving points in $\mathrm{R}^{d}$. If the $d+2$ points lie on a common $(d-1)$-sphere $\mathcal{S}$ at time $t$, then the points can be described by the equations

$$
\begin{aligned}
& x_{i}=c+r \cdot u_{i}-t \cdot v_{i}, \\
& y_{i}=x_{i}+v_{i},
\end{aligned}
$$

where $c \in \mathrm{R}^{d}$ is the center of $\mathcal{S}, r$ is its radius, $u_{i} \in \sigma_{d-1}$ is the unit vector $\left(x_{i}-c\right) /\left|x_{i}-c\right|$ describing $P_{i}$ 's position on $\mathcal{S}$, and $v_{i}=y_{i}-x_{i}$ represents the travel of $P_{i}$. 
Our goal is to use these equations to express the density

$$
\bigwedge_{i=1}^{d+2} d \lambda^{2}\left(P_{i}\right)=\bigwedge_{i=1}^{d+2}\left(d \lambda\left(x_{i}\right) \wedge d \lambda\left(y_{i}\right)\right)=\bigwedge_{i=1}^{d+2} \bigwedge_{j=1}^{d}\left(d x_{i}^{(j)} \wedge d y_{i}^{(j)}\right)
$$

in terms of the $d c^{(j)}, d r, d t, d u_{i}^{(j)}$, and $d v_{i}^{(j)}$.

From (2.2), we have

$$
\begin{aligned}
d x_{i}^{(j)} \wedge d y_{i}^{(j)} & =\left(d P_{i}^{(j)}-v_{i}^{(j)} d t\right) \wedge\left((1-t)^{-1} d y_{i}^{(j)}\right) \\
& =\left(d c^{(j)}+d\left(r u_{i}^{(j)}\right)-v_{i}^{(j)} d t\right) \wedge\left((1-t)^{-1} d y_{i}^{(j)}\right)
\end{aligned}
$$

Therefore, we have

$$
\bigwedge_{i=1}^{d+2} d \lambda^{2}\left(P_{i}\right)=\left(\bigwedge_{i=1}^{d+2} \bigwedge_{j=1}^{d}\left(d c^{(j)}+d\left(r u_{i}^{(j)}\right)-v_{i}^{(j)} d t\right)\right) \wedge\left(\bigwedge_{i=1}^{d+2}(1-t)^{-d} d \lambda\left(y_{i}\right)\right)
$$

It is a simple consequence of the axioms of the exterior algebra that, for any scalars $\alpha_{k}$ and differentials $d z_{k}$,

$$
\left(\sum_{k} \alpha_{k} d z_{k}\right) \wedge\left(\sum_{k} \alpha_{k} d z_{k}\right)=0
$$

In particular, for any fixed $i$ and $j$,

$$
\left(d c^{(j)}+d\left(r u_{i}^{(j)}\right)-v_{i}^{(j)} d t\right) \wedge\left(d c^{(j)}+d\left(r u_{i}^{(j)}\right)-v_{i}^{(j)} d t\right)=0 .
$$

Thus, if we define

$$
\Sigma_{i}=\sum_{k=1}^{d} u_{i}^{(k)}\left(d c^{(k)}+d\left(r u_{i}^{(k)}\right)-v_{i}^{(k)} d t\right)
$$

we see that, for any $i$,

$$
\begin{aligned}
\Sigma_{i} & \wedge \bigwedge_{j=2}^{d}\left(d c^{(j)}+d\left(r u_{i}^{(j)}\right)-v_{i}^{(j)} d t\right) \\
& =\sum_{k=1}^{d}\left(u_{i}^{(k)}\left(d c^{(k)}+d\left(r u_{i}^{(k)}\right)-v_{i}^{(k)} d t\right) \wedge \bigwedge_{j=2}^{d}\left(d c^{(j)}+d\left(r u_{i}^{(j)}\right)-v_{i}^{(j)} d t\right)\right) \\
& =u_{i}^{(1)}\left(d c^{(1)}+d\left(r u_{i}^{(1)}\right)-v_{i}^{(1)} d t\right) \wedge \bigwedge_{j=2}^{d}\left(d c^{(j)}+d\left(r u_{i}^{(j)}\right)-v_{i}^{(j)} d t\right) \\
& =u_{i}^{(1)} \bigwedge_{j=1}^{d}\left(d c^{(j)}+d\left(r u_{i}^{(j)}\right)-v_{i}^{(j)} d t\right) .
\end{aligned}
$$


So

$$
\begin{aligned}
\bigwedge_{i=1}^{d+2} d \lambda^{2}\left(P_{i}\right)= & \left(\bigwedge_{i=1}^{d+2} \Sigma_{i}\right) \wedge\left(\bigwedge_{i=1}^{d+2} \frac{1}{u_{i}^{(1)}} \bigwedge_{j=2}^{d}\left(d c^{(j)}+d\left(r u_{i}^{(j)}\right)-v_{i}^{(j)} d t\right)\right) \\
& \wedge\left(\bigwedge_{i=1}^{d+2}(1-t)^{-d} d \lambda\left(y_{i}\right)\right) .
\end{aligned}
$$

Since

$$
\left\langle u_{i}, d\left(r u_{i}\right)\right\rangle=r\left\langle u_{i}, d u_{i}\right\rangle+\left\langle u_{i}, u_{i}\right\rangle d r=r \cdot 1 \cdot d(1)+1 \cdot d r=d r
$$

we have

$$
\begin{aligned}
\Sigma_{i} & =\left\langle u_{i},\left(d c+d\left(r u_{i}\right)-v_{i} d t\right)\right\rangle \\
& =\left\langle u_{i}, d c\right\rangle+d r-\left\langle u_{i}, v_{i}\right\rangle d t .
\end{aligned}
$$

Thus, $\bigwedge_{i=1}^{d+2} \Sigma_{i}=\operatorname{det}(U) d c^{(1)} \wedge \cdots \wedge d c^{(d)} \wedge d r \wedge d t$, where $U$ is the matrix whose $i$ th row is

$$
\left(u_{i}^{(1)}, u_{i}^{(2)}, \ldots, u_{i}^{(d)},\left\langle u_{i}, v_{i}\right\rangle, 1\right)
$$

and, by applying first the cancellation rule $d z \wedge d z=0$ and then (2.1), we obtain

$$
\begin{aligned}
\bigwedge_{i=1}^{d+2} d \lambda^{2}\left(P_{i}\right) \\
=\operatorname{det}(U) d c^{(1)} \cdots d c^{(d)} d r d t\left(\bigwedge_{i=1}^{d+2} \frac{1}{u_{i}^{(1)}} \bigwedge_{j=2}^{d}\left(d c^{(j)}+d\left(r u_{i}^{(j)}\right)-v_{i}^{(j)} d t\right)\right) \\
\quad \wedge\left(\bigwedge_{i=1}^{d+2}(1-t)^{-d} d \lambda\left(y_{i}\right)\right) \\
\left.=\operatorname{det}(U) d c^{(1)} \cdots d c^{(d)} d r d t \bigwedge_{i=1}^{d+2} \frac{1}{u_{i}^{(1)}} \bigwedge_{j=2}^{d} d\left(r u_{i}^{(j)}\right)\right)\left(\bigwedge_{i=1}^{d+2}(1-t)^{-d} d \lambda\left(y_{i}\right)\right) \\
=\operatorname{det}(U) d \lambda(c) d r d t\left(\bigwedge_{i=1}^{d+2} r^{d-1} d \sigma\left(u_{i}\right)(1-t)^{-d} d \lambda\left(y_{i}\right)\right)
\end{aligned}
$$

Lastly, we express the center $c$ in terms of $q$, its distance from the origin, and unit vector $w=c /|c|$ :

$$
\bigwedge_{i=1}^{d+2} d \lambda^{2}\left(P_{i}\right)=q^{d-1} \operatorname{det}(U) d q d r d t d \sigma(w) \wedge\left(\bigwedge_{i=1}^{d+2} r^{d-1}(1-t)^{-d} d \sigma\left(u_{i}\right) d \lambda\left(y_{i}\right)\right) .
$$




\section{The Expected Number of Voronoi Diagram Transitions}

Let $\mathrm{E} T_{n}$ represent the expected number of Voronoi transitions of $\mathcal{P}_{n}$. Since the random moving points are i.i.d.,

$$
\begin{aligned}
\mathrm{E} T_{n} & =\left(\begin{array}{c}
n \\
d+2
\end{array}\right) \frac{\int\left(1-\varphi\left(P_{1}, \ldots, P_{d+2}\right)\right)^{n-d-2} d \lambda^{2}\left(P_{1}\right) \cdots d \lambda^{2}\left(P_{d+2}\right)}{\int d \lambda^{2}\left(P_{1}\right) \cdots d \lambda^{2}\left(P_{d+2}\right)} \\
& \sim \frac{n^{d+2}}{(d+2) !} B_{d}^{-2(d+2)} \int(1-\varphi)^{n-d-2} d \lambda^{2}\left(P_{1}\right) \cdots d \lambda^{2}\left(P_{d+2}\right),
\end{aligned}
$$

where integration is over all $(d+2)$-tuples of points moving in the $d$-ball, and $\varphi=$ $\varphi\left(P_{1}, \ldots, P_{d+2}\right)$ is defined as follows:

- If at some time $t \in[0,1]$, the $d+2$ random moving points $P_{1}, \ldots, P_{d+2}$ lie on the surface of a common $d$-ball, then $\varphi$ is the probability that a single random moving point lies inside that common $d$-ball at time $t$.

- If no such time $t$ exists, then $\varphi=1$.

Adopting the parameterization of the $d+2$ moving points described in Section 3, we obtain

$$
\begin{aligned}
\mathrm{E} T_{n} \sim & \frac{n^{d+2}}{(d+2) !} B_{d}^{-2(d+2)} \int(1-\varphi)^{n-d-2} \operatorname{det}(U) q^{d-1} \\
& \times d q d r d t d \sigma(w)\left(\bigwedge_{i=1}^{d+2} r^{d-1}(1-t)^{-d} d \sigma\left(u_{i}\right) d \lambda\left(y_{i}\right)\right) .
\end{aligned}
$$

Nothing else depends on $w$, and we may integrate $\int d \sigma_{d-1}(w)=d \cdot B_{d}$. The probability $\varphi\left(P_{1}, \ldots, P_{d+2}\right)$ depends only on $q, r$, and $t$, as do the integrals $\int r^{d-1}(1-$ $t)^{-d} d \sigma\left(u_{i}\right) d \lambda\left(y_{i}\right)$ for $1 \leq i \leq d+2$. In fact, for fixed $r, q$, and $t$, the quantity

$$
\frac{\int \operatorname{det}(U)\left(\bigwedge_{i=1}^{d+2} r^{d-1}(1-t)^{-d} d \sigma\left(u_{i}\right) d \lambda\left(y_{i}\right)\right)}{\int\left(\bigwedge_{i=1}^{d+2} r^{d-1}(1-t)^{-d} d \sigma\left(u_{i}\right) d \lambda\left(y_{i}\right)\right)}=: \mathrm{E}|U|(q, r, t)
$$

is the expected value of $\operatorname{det}(U)$ for $d+2$ random moving points that lie on sphere of radius $r$ centered at point $C$ at distance $q$ from the origin at time $t$. Since the $d+2$ moving points are i.i.d., the denominator is equal to

$$
\begin{aligned}
T(q, r, t)^{d+2} & :=\left(\int r^{d-1}(1-t)^{-d} d \sigma\left(u_{1}\right) d \lambda\left(y_{1}\right)\right)^{d+2} \\
& =\left(\int f_{t}\left(\left|C+r u_{1}\right|\right) r^{d-1} d \sigma\left(u_{1}\right)\right)^{d+2} \\
& \leq\left(f_{t}(\max (0, q-r)) r^{d-1} \int d \sigma\left(u_{1}\right)\right)^{d+2}
\end{aligned}
$$

the upper bound following from the monotonicity of $f_{t}$. 
For reasons of symmetry, we can restrict our attention to the interval $t \in\left[0, \frac{1}{2}\right]$. Finally, we can estimate $(1-\varphi(q, r, t))^{n-d-2}$ by $\exp (-n \varphi(q, r, t))$, and write

$$
\begin{aligned}
\mathrm{E} T_{n} \sim & \frac{2 d \cdot n^{d+2}}{(d+2) !} B_{d}^{-2 d-3} \\
& \times \int_{0}^{1 / 2} \int_{0}^{\infty} \int_{0}^{\infty} \exp (-n \varphi(q, r, t)) \mathrm{E}|U|(q, r, t) T(q, r, t)^{d+2} q^{d-1} d r d q d t .
\end{aligned}
$$

It is now only necessary to estimate $\varphi(q, r, t), \mathrm{E}|U|(q, r, t)$, and $T(q, r, t)$ for the random ball $\mathcal{S}$ defined by the $d+2$ random moving points.

Two crude but simple estimates are $T(q, r, t)=O(1)$ and $\mathrm{E}|U|(q, r, t)=O(1)$. (The bound for $T$ follows because the range of integration in (4.2) is closed and the integrand itself is $O(1)$. To bound $\mathrm{E}|U|$, we note that magnitudes of the entries in matrix $U$ are bounded: $\left|u_{i}^{(j)}\right| \leq 1$ and $\left|\left\langle u_{i}, v_{i}\right\rangle\right| \leq\left|u_{i}\right| \cdot\left|v_{i}\right| \leq 1 \cdot 2 \leq 2$.)

If $\varphi(q, r, t)=\Omega(1)$ over some subset of the range of integration, then the integrand approaches 0 exponentially as $n \rightarrow \infty$ and that subset's contribution is negligible. Thus, we may restrict our attention to $(d-1)$-spheres $\mathcal{S}$ with small intersections with the unit ball. In particular, we ignore intersections that contain any $d$-ball of radius $\frac{1}{8}$.

We distinguish three cases, depending on whether $\mathcal{S}$ lies entirely inside or partly outside the unit ball $(q+r \leq />1)$, and, in the latter case, if the center $C$ of $\mathcal{S}$ lies inside or outside the unit ball $(q \leq />1)$.

Case I: $q \leq 1-r$ and $r \leq \frac{1}{8}$. In this case, $\mathcal{S}$ lies entirely within the unit ball. (Since the contribution of this case dominates, the most careful estimates are required.)

Case Ia: $q \leq \frac{1}{8}$. In this case,

$$
\begin{aligned}
r^{d} \min _{\tau \in[0,1 / 2]} f_{\tau}\left(\frac{1}{8}\right) & \leq \varphi(q, r, t) \leq r^{d} f_{1 / 2}(0), \\
r^{d-1} \min _{\tau \in[0,1 / 2]} f_{\tau}\left(\frac{1}{8}\right) & \leq T(q, r, t) \leq r^{d-1} f_{1 / 2}(0),
\end{aligned}
$$

so $\varphi=\Theta\left(r^{d}\right), T=\Theta(\varphi / r)$, and $\mathrm{E}|U|=\Theta(1)$.

Case $I b: q \geq \frac{1}{8}$. First, we compute the set $\mathcal{Y}$ of destinations of moving points originating in the unit ball and passing through $C$ at time $t$. As in the proof of Lemma 2, this a lens with volume $\Upsilon:=L(|C| / t,(1-t) / t)=L(q / t,(1-t) / t)$. (We assume the subscript of $L$ is $d$ in this section.)

Now, the set of sources $\mathcal{X}_{Y}$ leading to a given destination $Y \in \mathcal{Y}$ after passing through $\mathcal{S}$ at time $t$ is also a $d$-ball with center somewhere in the unit ball (by definition of $\mathcal{Y}$ ) and radius $r /(1-t)$. (Specifically, the point's location $P=(1-t) X+t Y$ at time $t$ satisfies $\delta(P, C) \leq r$ if and only if $\delta(X+t Y /(1-t), C /(1-t))=\delta(P /(1-t), C /(1-t)) \leq$ $r /(1-t)$.) Since $r<\frac{1}{8}$ and $t<\frac{1}{2}, \mathcal{X}_{Y}$ is smaller than the unit ball. Since its center lies inside the unit ball, the intersection contains a ball of radius $r / 2$, and

$$
(r / 2)^{d}(1-t)^{-d} \leq \operatorname{Pr}\left(\mathcal{X}_{Y}\right) \leq r^{d}(1-t)^{-d},
$$

and $\operatorname{Pr}\left(\mathcal{X}_{Y}\right)=\Theta(\Xi)$ where $\Xi=r^{d}$. Thus, the probability that a random moving point 
passes through the $(q, r)$-ball at time $t$ is

$$
\varphi(q, r, t)=\Omega(\Xi \Upsilon)
$$

Next, we observe that

$$
\begin{aligned}
T(q, r, t) & \leq r^{d-1} f_{t}(q-r) \\
& \leq r^{d-1} L\left(\frac{q-r}{t}, \frac{1-t}{t}\right) \cdot \\
& =O(1) \cdot\left(\frac{\Xi}{r}\right) \cdot \Upsilon \cdot \frac{L((q-r) / t,(1-t) / t)}{L(q / t,(1-t) / t)} \\
& =O(1) \cdot\left(\frac{\Xi}{r}\right) \cdot \Upsilon \cdot \frac{\max \left(1,((1-q+r) / t)^{(d+1) / 2}\right)}{\max \left(1,((1-q) / t)^{(d+1) / 2}\right)} \\
& =O(1) \cdot\left(\frac{\Xi \Upsilon}{r}\right) \cdot 2^{(d+1) / 2} \\
& =O\left(\frac{\Xi \Upsilon}{r}\right)
\end{aligned}
$$

since $1-q+r \leq 2(1-q)$.

Also, $\mathrm{E}|U|(q, r, t)=\Theta(1)$.

So for Cases Ia and Ib together, we have a contribution of

$$
\begin{aligned}
\mathrm{E} T_{n}^{(\mathrm{I})} & =O\left(n^{d+2}\right) \int_{0}^{1 / 2} \int_{0}^{1 / 8} \int_{0}^{1-r} e^{-n \varphi}\left(\frac{\varphi}{r}\right)^{d+2} q^{d-1} d q d r d t \\
& =O(1) \int_{0}^{1 / 2} \int_{0}^{\infty} \int_{0}^{1} e^{-u}\left(\frac{u}{r}\right)^{d+1} q^{d-1} d q d u d t \\
& =O(1) \int_{0}^{1 / 2} \int_{0}^{\infty} \int_{0}^{1} e^{-u} u^{d+1}\left(\frac{u}{n L(q / t,(1-t) / t)}\right)^{-(d+1) / d} q^{d-1} d q d u d t \\
& =O\left(n^{(d+1) / d}\right)\left(\int_{0}^{1 / 2} d t\right)\left(\int_{0}^{\infty} e^{-u} u^{d-(1 / d)} d u\right)\left(\int_{0}^{1} q^{d-1} d q\right) \\
& =O\left(n^{(d+1) / d}\right)
\end{aligned}
$$

where we have set $u=n \varphi, d u=\Theta(u / r) d r$, and $r=(u /(n L(q / t,(1-t) / t)))^{1 / d}$, and observed that $L(q / t,(1-t) / t)=O(1)$.

Case II: $1-r \leq q \leq 1$ and $r \leq \frac{1}{8}$. In this case the center of $\mathcal{S}$ lies inside the unit ball, although part also lies outside. We have

$$
\begin{aligned}
\varphi(q, r, t) & \geq \varphi(1-(r / 2), r / 2, t)=\Omega\left(r^{d} L((1-(r / 2)) / t,(1-t) / t)\right) ; \\
T(q, r, t) & \leq T(1-r, r, t) \leq r^{d-1} f_{t}(1-r) \\
& =O\left(r^{d-1} L((1-r) / t,(1-t) / t)\right)=O(\varphi / d) ; \\
\mathrm{E}|U|(q, r, t) & =O(1) .
\end{aligned}
$$


Thus

$$
\begin{aligned}
\mathrm{E} T_{n}^{(\mathrm{II})} & =O\left(n^{d+2}\right) \int_{0}^{1 / 2} \int_{0}^{1 / 8} \int_{1-r}^{1} e^{-n \varphi} \mathrm{E}|U| T^{d+2} q^{d-1} d q d r d t \\
& =O(1) \int_{0}^{1 / 2} \int_{0}^{1 / 8} \int_{1-r}^{1} e^{-n \varphi}(n \varphi / r)^{d+2} d q d r d t \\
& =O(1) \int_{0}^{1 / 2} \int_{0}^{1 / 8} e^{-n \varphi}(n \varphi)^{d+2} r^{-(d+1)} d r d t \\
& =O(1) \int_{0}^{1 / 2} \int_{0}^{\infty} e^{-u} u^{d+2} r^{-(d+1)}(r / u) d u d t \\
& =O(1) \int_{0}^{1 / 2} \int_{0}^{\infty} e^{-u} u^{d+1} r^{-d} d u d t
\end{aligned}
$$

where $u=n \varphi=n r^{d} L((1-r) / t,(1-t) / t)$ and $d u=O(u / r) d r$. Thus $r^{-d}=$ $(n / u) L((1-r) / t,(1-t) / t)=O(n / u)$ and

$$
\mathrm{E} T_{n}^{(\mathrm{II})}=O(n)\left(\int_{0}^{1 / 2} d t\right)\left(\int_{0}^{\infty} e^{-u} u^{d} d u\right)=O(n)
$$

Case III: $q>1$ and $q-1 \leq r \leq q-\frac{7}{8}$. We proceed roughly as for Case I, but we let $C$ be the center of the lens formed by the intersection of $\mathcal{S}$ with the unit ball. Let $w=1-q+r$ be the thickness of the lens, and let $h$ be its radius (Fig. 1). The volume of the lens is $\Theta\left(w h^{d-1}\right)$, and $|C|=1-(w / 2)=(2-w) / 2$. By routine application of the Pythagorean Theorem, we can show that

$$
x=\frac{r^{2}+q^{2}-1}{2 q}=r-\Theta\left(\frac{w}{q}\right)
$$

and

$$
h=\frac{1}{2 q} \sqrt{-(1+q+r)(1+q-r)(1-q+r)(1-q-r)}=\Theta\left(\sqrt{\frac{w r}{q}}\right) .
$$

The set $\mathcal{Y}$ of destinations of moving points originating in the unit ball and passing through

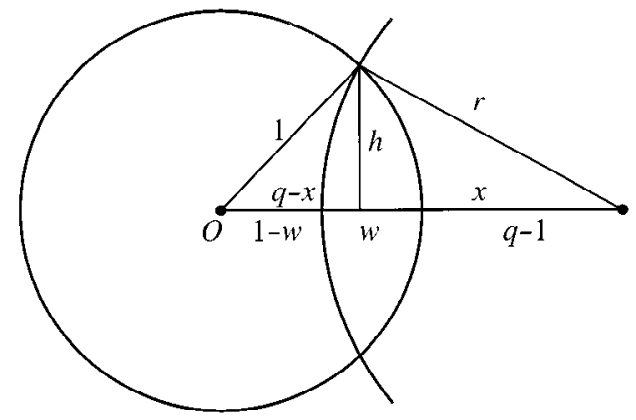

Fig. 1. Case III. 
$C$ at time $t$ is the intersection of the unit ball with a ball with center $(2-w) /(2 t)$ and radius $(1-t) / t$. So $\lambda(\mathcal{Y})=\Upsilon:=L((2-w) /(2 t),(1-t) / t)$.

For each such destination $Y \in \mathcal{Y}$, the corresponding sources that pass through the lens at time $t$ lie in a projected lens of volume $\Theta(\Xi)$, where $\Xi:=w h^{d-1}(1-t)^{-d}$. At least a constant fraction of this projected lens lies inside the unit ball. Therefore, $\lambda\left(\mathcal{X}_{Y}\right)=\Omega(\Xi)$, and

$$
\varphi(q, r, t)=\Omega\left(\left(\min _{Y \in \mathcal{Y}} \lambda\left(\mathcal{X}_{Y}\right)\right) \lambda(\mathcal{Y})\right)=\Omega(\Xi \Upsilon) .
$$

The portion of the surface of $\mathcal{S}$ lying inside the unit ball has area at least $B_{d-1} h^{d-1}$ and at most $\left(S_{d-1} / 2\right) h^{d-1}$. Thus, by (4.2),

$$
T=O\left(h^{d-1} f_{t}(q-r)\right)=O\left(h^{d-1} L((2-w) /(2 t),(1-t) / t)\right)=O(\Xi \Upsilon / w) .
$$

The determinant $|U|$ is $r^{-d}$ times the determinant of the matrix whose $i$ th row is

$$
\left(r u_{i}^{(1)}, r u_{i}^{(2)}, \ldots, r u_{i}^{(d)},\left\langle u_{i}, v_{i}\right\rangle, 1\right)
$$

or, equivalently,

$$
\left(x_{i}^{(1)}, x_{i}^{(2)}, \ldots, x_{i}^{(d)},\left\langle u_{i}, v_{i}\right\rangle, 1\right) .
$$

However, this is just $(d+1)$ ! times the volume of a simplex in $\mathrm{R}^{d+1}$ contained in the Cartesian product of the inner half of the lens and the interval $[-2,2]$. Thus,

$$
\mathrm{E}|U| \leq r^{-d} \cdot(r-x) h^{d-1} \cdot 4=O\left(\Xi q^{-1} r^{-d}\right)
$$

So

$$
\begin{aligned}
\mathrm{E} T_{n}^{(\mathrm{III})}= & O\left(n^{d+2}\right) \int_{0}^{1 / 2} \int_{1}^{\infty} \int_{q-1}^{q-7 / 8} \exp (-n \Omega(\Xi \Upsilon))\left(\Xi q^{-1} r^{-d}\right)\left(\Xi \Upsilon w^{-1}\right)^{d+2} q^{d-1} \\
& \times d r d q d t \\
= & O\left(n^{d+2}\right) \int_{0}^{1 / 2} \int_{1}^{\infty} \int_{0}^{1 / 8} \exp (-n \Omega(\Xi \Upsilon))(\Xi \Upsilon)^{d+1} w^{-(d+2)} \Xi^{2} \Upsilon q^{d-2} r^{-d} \\
& \times d w d q d t \\
= & O\left(n^{d+2}\right) \int_{0}^{1 / 2} \int_{1}^{\infty} \int_{0}^{1 / 8} \exp (-n \Omega(\Xi \Upsilon))(\Xi \Upsilon)^{d+1} \Upsilon q^{-1} r^{-1} w^{-1} d w d q d t \\
= & O\left(n^{d+2}\right) \int_{0}^{1 / 2} \int_{1}^{\infty} \int_{0}^{1 / 8} \exp (-n \Omega(\Xi \Upsilon))(\Xi \Upsilon)^{d+1-1 /(d+1)} \Xi^{1 /(d+1)} \\
& \times \Upsilon^{(d+2) /(d+1)} q^{-1} r^{-1} w^{-1} d w d q d t \\
= & O\left(n^{d+2}\right) \int_{0}^{1 / 2} \int_{1}^{\infty} \int_{0}^{\infty} e^{-u}(u / n)^{d+1-1 /(d+1)} \Xi^{1 /(d+1)} q^{-1} r^{-1} u^{-1} d u d q d t \\
= & O\left(n^{(d+2) /(d+1)}\right)\left(\int_{0}^{\infty} e^{-u} u^{d-1 /(d+1)} d u\right)\left(\int_{0}^{1 / 2} d t\right)\left(\int_{1}^{\infty} \Xi^{1 /(d+1)} q^{-1} r^{-1} d q\right) .
\end{aligned}
$$


Thus $\mathrm{E} T_{n}^{(\mathrm{III})}=o\left(n^{(d+1) / d}\right)$ if each of the three integrals is $O(1)$. The first is equal to $\Gamma(d+1-1 /(d+1))=O(1)$. The second is equal to $\frac{1}{2}$. To bound the third integral, we note that $r^{-1 / 2} \leq w^{-1 / 2}$ and $r^{-1 / 2} \leq(q-1)^{-1 / 2}$ because $r \geq w$ and $r \geq q-1$. So

$$
\Xi^{1 /(d+1)}=\left[w\left(\frac{w r}{q}\right)^{(d-1) / 2}\right]^{1 /(d+1)}=w^{1 / 2}\left(\frac{r}{q}\right)^{(d-1) /(2 d+2)} \leq w^{1 / 2}
$$

Therefore,

$$
\begin{aligned}
\int_{1}^{\infty} \Xi^{1 /(d+1)} q^{-1} r^{-1} d q & \leq \int_{1}^{\infty} w^{1 / 2} q^{-1}\left((q-1)^{-1 / 2} w^{-1 / 2}\right) d q \\
& =\int_{1}^{\infty} q^{-1}(q-1)^{-1 / 2} d q=\pi \approx 3.14
\end{aligned}
$$

Finally, summing over all three cases gives $\mathrm{E} T_{n}=O\left(n^{(d+1) / d}\right)$.

\section{A Density Transformation for Convex Hulls}

Let $P_{i}=P_{i}\left(x_{i}, y_{i}\right)$ for $1 \leq i \leq d+1$ be the initial and final positions of $d+1$ moving points in $\mathrm{R}^{d}$. If the $d+1$ points lie on a common hyperplane $\mathcal{H}$ at time $t$, then the points can be described by the equations

$$
\begin{aligned}
P_{i} & =(1-t) x_{i}+t y_{i}, \\
P_{i} & =c+Q_{i}=c+\sum_{j=1}^{d} q_{i}^{(j)} e_{j}, \quad \text { and } \\
y_{i} & =x_{i}+v_{i},
\end{aligned}
$$

where $c \in \mathrm{R}^{d}$ is the point on $\mathcal{H}$ lying nearest the origin (the "foot" of $\mathcal{H}$ ), and $e_{1}, e_{2}, \ldots, e_{d-1} \in \mathrm{R}^{d}$ form an orthonormal basis for $\mathcal{H}$. (That is, $\left\langle e_{i}, e_{j}\right\rangle=\delta_{i j}$ and $\left\langle c, e_{i}\right\rangle=0$ for all $i$ and $j$.)

From (2.2), we have

$$
d x_{i}^{(j)} \wedge d y_{i}^{(j)}=\left(d P_{i}^{(j)}-v_{i}^{(j)} d t\right) \wedge\left((1-t)^{-1} d y_{i}^{(j)}\right) .
$$

Setting

$$
\begin{aligned}
\Sigma_{i} & =\left(1 / c^{(1)}\right)\left\langle c,\left(d P_{i}-v_{i} d t\right)\right\rangle \\
& =\left(1 / c^{(1)}\right)\left(\left\langle c, d P_{i}\right\rangle-\left\langle c, v_{i}\right\rangle d t\right) \\
& =\left(1 / c^{(1)}\right)\left(d\left\langle c, P_{i}\right\rangle-\left\langle P_{i}, d c\right\rangle-\left\langle c, v_{i}\right\rangle d t\right) \\
& =\left(1 / c^{(1)}\right)\left(d\langle c, c\rangle-\left\langle P_{i}, d c\right\rangle-\left\langle c, v_{i}\right\rangle d t\right) \\
& =\left(1 / c^{(1)}\right)\left(2\langle c, d c\rangle-\left\langle P_{i}, d c\right\rangle-\left\langle c, v_{i}\right\rangle d t\right) \\
& =\left(1 / c^{(1)}\right)\left(\left\langle 2 c-P_{i}, d c\right\rangle-\left\langle c, v_{i}\right\rangle d t\right),
\end{aligned}
$$

we find that $\bigwedge_{i=1}^{d+1} \Sigma_{i}=\left(c^{(1)}\right)^{-(d+1)} \operatorname{det}(M) d \lambda(c) d t$, where $M$ is the matrix whose $i$ th row is

$$
\left(2 c^{(1)}-P_{i}^{(1)}, 2 c^{(2)}-P_{i}^{(2)}, \ldots, 2 c^{(d)}-P_{i}^{(d)},\left\langle c, v_{i}\right\rangle\right) .
$$


However, this matrix is $(d+1)$ ! times the $(d+1)$-volume of the $(d+1)$-simplex formed by the origin and the $(d+1)$ row-vectors of $M$, or, setting $u=c /|c|$ and translating the origin, $2|c|^{2}(d+1)$ ! times the $d$-volume of the $d$-simplex formed on $\mathcal{H} \times \mathrm{R}$ by the points

$$
\left(P_{i}^{(1)}, P_{i}^{(2)}, \ldots, P_{i}^{(d)},\left\langle u, v_{i}\right\rangle\right)
$$

a $d$-volume that we denote by $\lambda(\triangle)$.

Now we have

$$
\begin{aligned}
\bigwedge_{i=1}^{d+1} d & \lambda^{2}\left(P_{i}\right) \\
= & \left(\bigwedge_{i=1}^{d+1} \Sigma_{i}\right) \wedge\left(\bigwedge_{i=1}^{d+1} \bigwedge_{j=2}^{d}\left(d P^{(j)}-v_{i}^{(j)} d t\right)\right) \wedge\left(\bigwedge_{i=1}^{d+1}(1-t)^{-d} d \lambda\left(y_{i}\right)\right) \\
= & \left.2|c|^{2}(d+1) !\left(c^{(1)}\right)^{-(d+1)} \lambda(\triangle) d \lambda(c) d t \bigwedge_{i=1}^{d+1} \bigwedge_{j=2}^{d}\left(d P_{i}^{(j)}-v_{i}^{(j)} d t\right)\right) \\
& \wedge\left(\bigwedge_{i=1}^{d+1}(1-t)^{-d} d \lambda\left(y_{i}\right)\right) \\
& \wedge\left(\bigwedge_{i=1}^{d+1}(1-t)^{-d} d \lambda\left(y_{i}\right)\right) \\
= & 2|c|^{2}(d+1) !\left(c^{(1)}\right)^{-(d+1)} \lambda(\triangle) d \lambda(c) d t\left(\bigwedge_{i=1}^{d+1} \bigwedge_{j=2}^{d} \sum_{k=1}^{d-1} e_{k}^{(j)} d q_{i}^{(k)}\right) \\
& \wedge\left(\bigwedge_{i=1}^{d+1}(1-t)^{-d} d \lambda\left(y_{i}^{(1)}\right)\right),
\end{aligned}
$$

the $d e_{k}^{(j)}$ terms being cancelled by $d \lambda(c)$ because the $e_{i}$ depend only on $c$. Now

$$
\bigwedge_{j=2}^{d} \sum_{k=1}^{d-1} e_{k}^{(j)} d q_{i}^{(k)}=\left|\begin{array}{llll}
e_{1}^{(2)} & e_{2}^{(2)} & \cdots & e_{d-1}^{(2)} \\
e_{1}^{(3)} & e_{2}^{(3)} & \cdots & e_{d-1}^{(3)} \\
\vdots & \vdots & \ddots & \vdots \\
e_{1}^{(d)} & e_{2}^{(d)} & \cdots & e_{d-1}^{(d)}
\end{array}\right| \bigwedge_{j=2}^{d} d q_{i}^{(j)}=\frac{c^{(1)}}{|c|} d \lambda_{d-1}\left(Q_{i}\right)
$$

since the prism formed by the orthonormal vectors $e_{i}$ is a unit $(d-1)$-cube, and the determinant represents the volume of the projection of this cube onto the plane $x^{(1)}=0$. Thus, with $|c|=r$, we have

$$
\bigwedge_{i=1}^{d+1} d \lambda^{2}\left(P_{i}\right)=2(d+1) ! \lambda(\triangle) r^{-(d-1)} d \lambda(c) d t\left(\bigwedge_{i=1}^{d+1}(1-t)^{-d} d \lambda\left(y_{i}\right) d \lambda_{d-1}\left(Q_{i}\right)\right) .
$$




\section{The Expected Number of Convex Hull Transitions}

Let $\mathrm{E} T_{n}$ represent the expected number of convex-hull transitions of $\mathcal{P}_{n}$. Since the random moving points are i.i.d.,

$$
\begin{aligned}
\mathrm{E} T_{n} & =\left(\begin{array}{c}
n \\
d+1
\end{array}\right) \frac{\int\left(1-\Gamma\left(P_{1}, \ldots, P_{d+1}\right)\right)^{n-d-1} d \lambda^{2}\left(P_{1}\right) \cdots d \lambda^{2}\left(P_{d+1}\right)}{\int d \lambda^{2}\left(P_{1}\right) \cdots d \lambda^{2}\left(P_{d+1}\right)} \\
& \sim \frac{n^{d+1}}{(d+1) ! B_{d}^{2(d+1)}} \int(1-\Gamma)^{n-d-1} d \lambda^{2}\left(P_{1}\right) \cdots d \lambda^{2}\left(P_{d+1}\right),
\end{aligned}
$$

where integration is over all $(d+1)$-tuples of points moving in the $d$-ball, and $\Gamma=$ $\Gamma\left(P_{1}, \ldots, P_{d+1}\right)$ is defined as follows:

- If at some time $t \in[0,1]$, the $d+1$ random moving points $P_{1}, \ldots, P_{d+1}$ lie on a common hyperplane $\mathcal{H}$, then $\Gamma$ is the probability that a single random moving point lies beyond $\mathcal{H}$ at time $t$.

- If no such time $t$ exists, then $\Gamma=1$.

Adopting the parameterization of the $d+1$ moving points described in Section 5, we obtain

$\mathrm{E} T_{n}=\Theta\left(n^{d+1}\right) \int(1-\Gamma)^{n-d-1} \lambda(\triangle)\left(\bigwedge_{i=1}^{d+1}(1-t)^{-d} d \lambda\left(y_{i}\right) d \lambda_{d-1}\left(Q_{i}\right)\right) r^{-(d-1)} d \lambda(c) d t$

The probability $\Gamma\left(P_{1}, \ldots, P_{d+1}\right)$ and the integrals $\int(1-t)^{-d} d \lambda\left(y_{i}\right) d \lambda_{d-1}\left(Q_{i}\right)$ for $1 \leq i \leq d+1$ depend only on $c$ and $t$. In fact, for fixed $c$ and $t$, the quantity

$$
\frac{\int \lambda(\triangle)\left(\bigwedge_{i=1}^{d+1}(1-t)^{-d} d \lambda\left(y_{i}\right) d \lambda_{d-1}\left(Q_{i}\right)\right)}{\int\left(\bigwedge_{i=1}^{d+1}(1-t)^{-d} d \lambda\left(y_{i}\right) d \lambda_{d-1}\left(Q_{i}\right)\right)}=: \mathrm{E} \triangle(c, t)
$$

is the expected value of $\lambda(\triangle)$ for $d+1$ random moving points that lie on the hyperplane with foot $c$ at time $t$. Since the $d+1$ moving points are i.i.d., the denominator is equal to

$$
\left(\int(1-t)^{-d} d \lambda\left(y_{i}\right) d \lambda_{d-1}\left(Q_{i}\right)\right)^{d+1}=: G(c, t)^{d+1} .
$$

For reasons of symmetry, we can restrict our attention to the interval $t \in\left[0, \frac{1}{2}\right]$. Finally, we can estimate $(1-\Gamma(c, t))^{n-d-1}$ by $\exp (-n \Gamma(c, t))$, and write

$$
\mathrm{E} T_{n}=\Theta\left(n^{d+1}\right) \int \exp (-n \Gamma(c, t)) G(c, t)^{d+1} \mathrm{E} \triangle(c, t) r^{-(d-1)} d \lambda(c) d t
$$

It is now only necessary to estimate $\Gamma(c, t), \mathrm{E} \triangle(c, t)$, and $G(c, t)$ for the hyperplane $\mathcal{H}=\mathcal{H}_{\perp c}$ defined by the $d+1$ random moving points. We base these estimates on another point, $x=x(c)$, lying on $\mathcal{H}$. Our first task is to relate $c$ to $x$ and $d \lambda(c)$ to $d \lambda(x)$.

We take the vector

$$
c=(r_{1}, \underbrace{0, \ldots, 0}_{d_{1}-1}, r_{2}, \underbrace{0, \ldots, 0}_{d_{2}-1}, \ldots, r_{k}, \underbrace{0, \ldots, 0}_{d_{k}-1})
$$


as representative of the class satisfying

$$
\sum_{j=j_{i}}^{j_{i}+d_{i}-1}\left(x^{(j)}\right)^{2}=r_{i}^{2} \quad \text { for } \quad i: 1 \leq i \leq k,
$$

where $j_{i}=1+\sum_{m=1}^{i-1} d_{m}$ is the index of the first coordinate in the $i$ th component ball. It is clear that estimates of $\Gamma, G$, and $\mathrm{E} \triangle$ for a representative are valid for its entire class. We define

$$
\begin{gathered}
x=(\left(1-y_{1}\right), \underbrace{0, \ldots, 0}_{d_{1}-1},\left(1-y_{2}\right), \underbrace{0, \ldots, 0}_{d_{2}-1}, \ldots,\left(1-y_{k}\right), \underbrace{0, \ldots, 0}_{d_{k}-1}), \\
\mathcal{H}_{x}=\left\{x \mid \sum_{i=1}^{k} \frac{1-x^{\left(j_{i}\right)}}{y_{i}}=k\right\}, \quad \text { and } \mathcal{H}_{x}^{+}=\left\{x \mid \sum_{i=1}^{k} \frac{1-x^{\left(j_{i}\right)}}{y_{i}} \leq k\right\} .
\end{gathered}
$$

It is clear from (6.4) that if $\mathcal{H}_{x}=\mathcal{H}_{\perp c}$, then

$$
\begin{gathered}
c /|c|=\left(\sum_{i=1}^{k} y_{i}^{-2}\right)^{-1 / 2} \cdot(y_{1}^{-1}, \underbrace{0, \ldots, 0}_{d_{1}-1}, y_{2}^{-1}, \underbrace{0, \ldots, 0}_{d_{2}-1}, \ldots, y_{k}^{-1}, \underbrace{0, \ldots, 0}_{d_{k}-1}), \\
|c|=\langle c /|c|, x\rangle=\left(\sum_{i=1}^{k} y_{i}^{-2}\right)^{-1 / 2}\left(\sum_{i=1}^{k} y_{i}^{-1}-k\right)=Y_{2}^{-1 / 2}\left(Y_{1}-k\right),
\end{gathered}
$$

and

$$
r_{i}=y_{i}^{-1} Y_{2}^{-1}\left(Y_{1}-k\right)
$$

For our particular distribution, we expand

$$
\begin{aligned}
d \lambda(c) & =\bigwedge_{i=1}^{k} r_{i}^{d_{i}-1} d r_{i} d \sigma\left(u_{i}\right) \\
& =Y_{2}^{-(d-k)}\left(Y_{1}-k\right)^{d-k} \bigwedge_{i=1}^{k} y_{i}^{-\left(d_{i}-1\right)} d r_{i} d \sigma\left(u_{i}\right) .
\end{aligned}
$$

Defining $\alpha=Y_{2}^{-1}\left(Y_{1}-k\right)$ and $\alpha_{i}^{\prime}=\partial \alpha / \partial y_{i}$, we have

$$
\begin{aligned}
d r_{i} & =y_{i}^{-1} d \alpha+\alpha d\left(y_{i}^{-1}\right) \\
& =y_{i}^{-1}\left(d \alpha-r_{i} d y_{i}\right) \\
& =y_{i}^{-1}\left(\sum_{j=1}^{k} \alpha_{j}^{\prime} d y_{j}-r_{i} d y_{i}\right)
\end{aligned}
$$

and

$$
\bigwedge_{i=1}^{k} d r_{i}=\left(\bigwedge_{i=1}^{k} y_{i}^{-1} d y_{i}\right) \cdot D
$$


where

$$
\begin{aligned}
D & =\left|\begin{array}{cccc}
\alpha_{1}^{\prime}-r_{1} & \alpha_{2}^{\prime} & \cdots & \alpha_{k}^{\prime} \\
\alpha_{1}^{\prime} & \alpha_{2}^{\prime}-r_{2} & \cdots & \alpha_{k}^{\prime} \\
\vdots & \vdots & \ddots & \vdots \\
\alpha_{1}^{\prime} & \alpha_{2}^{\prime} & \cdots & \alpha_{k}^{\prime}-r_{k}
\end{array}\right|=\left|\begin{array}{ccccc}
1 & -\alpha_{1}^{\prime} & -\alpha_{2}^{\prime} & \cdots & -\alpha_{k}^{\prime} \\
1 & -r_{1} & 0 & \cdots & 0 \\
1 & 0 & -r_{2} & \cdots & 0 \\
\vdots & \vdots & \vdots & \ddots & \vdots \\
1 & 0 & 0 & \cdots & -r_{k}
\end{array}\right| \\
& =(-1)^{k} r_{1} r_{2} \cdots r_{k}\left(1-\sum_{i=1}^{k} \frac{\alpha_{i}^{\prime}}{r_{i}}\right)
\end{aligned}
$$

Now

$$
\begin{aligned}
1-\sum_{i=1}^{k} \frac{\alpha_{i}^{\prime}}{r_{i}} & =1-\sum_{i=1}^{k} \frac{y_{i}}{\alpha} \cdot \frac{\partial \alpha}{\partial y_{i}} \\
& =1-\sum_{i=1}^{k} y_{i} \cdot \frac{\partial}{\partial y_{i}} \log (\alpha) \\
& =1-\sum_{i=1}^{k} y_{i}\left[\frac{\partial}{\partial y_{i}} \log \left(Y_{1}-k\right)-\frac{\partial}{\partial y_{i}} \log \left(Y_{2}\right)\right] \\
& =1-\sum_{i=1}^{k} y_{i}\left[\left(Y_{1}-k\right)^{-1} \frac{\partial}{\partial y_{i}}\left(Y_{1}-k\right)-Y_{2}^{-1} \frac{\partial}{\partial y_{i}}\left(Y_{2}\right)\right] \\
& =1-\sum_{i=1}^{k} y_{i}\left[\left(Y_{1}-k\right)^{-1}\left(-y_{i}^{-2}\right)-Y_{2}^{-1}\left(-2 y_{i}^{-3}\right)\right] \\
& =1-\sum_{i=1}^{k}\left[2 Y_{2}^{-1} y_{i}^{-2}-\left(Y_{1}-k\right)^{-1}\left(y_{i}^{-1}\right)\right] \\
& =1-\left[2 Y_{2}^{-1} Y_{2}-\left(Y_{1}-k\right)^{-1} Y_{1}\right] \\
& =k\left(Y_{1}-k\right)^{-1} .
\end{aligned}
$$

Combining this with (6.5)-(6.7), we have

$$
\begin{aligned}
d \lambda(c) & =\frac{k}{Y_{1}-k} \alpha^{d}\left(\prod_{i=1}^{k} y_{i}^{-\left(d_{i}+1\right)}\right) \bigwedge_{i=1}^{k}\left(d y_{i} \wedge d \sigma\left(u_{i}\right)\right) \\
& =k r^{d-1} Y_{2}^{-(d+1) / 2}\left(\prod_{i=1}^{k} y_{i}^{-\left(d_{i}+1\right)}\right) \bigwedge_{i=1}^{k}\left(d y_{i} \wedge d \sigma\left(u_{i}\right)\right)
\end{aligned}
$$

and, from (6.3),

$$
\begin{aligned}
\mathrm{E} T_{n} & =\Theta\left(n^{d+1}\right) \int e^{-n \Gamma} G^{d+1} \mathrm{E} \triangle k Y_{2}^{-(d+1) / 2}\left(\prod_{i=1}^{k} y_{i}^{-\left(d_{i}+1\right)}\right) d t \bigwedge_{i=1}^{k} d y_{i} d \sigma_{d_{i}-1}\left(u_{i}\right) \\
& =\Theta\left(n^{d+1}\right) \int e^{-n \Gamma} G^{d+1} \mathrm{E} \triangle Y_{2}^{-(d+1) / 2}\left(\prod_{i=1}^{k} y_{i}^{-\left(d_{i}+1\right)}\right) d t d y_{1} \cdots d y_{k} .
\end{aligned}
$$


For our remaining estimates, we need an asymptotically tight bound on the volume $V$ of $\mathcal{H}_{x}^{+} \cap \mathcal{K}$. To obtain an upper bound, we note that $V$ is proportional to

$$
V=\Theta(1) \int \cdots \int u_{1}^{\left(d_{1}-1\right) / 2} d u_{1} \cdots u_{k}^{\left(d_{k}-1\right) / 2} d u_{k}
$$

with integration over the region

$$
\sum_{i=1}^{k} \frac{u_{i}}{y_{i}} \leq k, \quad 0 \leq u_{i} \leq 1 \quad \text { for } \quad 1 \leq i \leq k .
$$

An upper bound is more easily obtained if the conditions $u_{i} \leq 1$ are ignored. If the innermost integration is carried out, the result is

$$
V=O(1) \cdot y_{1}^{\left(d_{1}+1\right) / 2}\left(k-\sum_{i=2}^{k} \frac{u_{i}}{y_{i}}\right)^{\left(d_{1}+1\right) / 2}
$$

making the dependence on $y_{1}$ explicit. However, since any of the integrations could be carried out first, this suffices for the asymptotic upper bound $V=O\left(\prod_{i=1}^{k} y_{i}^{\left(d_{i}+1\right) / 2}\right)$. On the other hand, this half-space also contains the set

$$
\mathcal{L}=\left\{x \in \mathcal{K} \mid x^{\left(j_{i}\right)} \geq 1-y_{i} \text { for all } 1 \leq i \leq k\right\},
$$

the Cartesian product of $k$ segments each having volume $\Omega\left(y^{\left(d_{i}+1\right) / 2}\right)$, so the corresponding asymptotic lower bound holds as well, and we have

$$
V=\Theta\left(\prod_{i=1}^{k} y_{i}^{\left(d_{i}+1\right) / 2}\right)
$$

To bound $\mathrm{E} \triangle\left(\mathcal{H}_{x}\right)$, we note first that the surface area $\mathcal{H}_{x} \cap \mathcal{K}$ is $O(V / \eta)$, where

$$
\begin{aligned}
\eta & =\left\langle c /|c|,(1, \underbrace{0, \ldots, 0}_{d_{1}-1}, 1, \underbrace{0, \ldots, 0}_{d_{2}-1}, \ldots, 1, \underbrace{0, \ldots, 0}_{d_{k}-1})\right\rangle-|c| \\
& =Y_{2}^{-1 / 2} Y_{1}-Y_{2}^{-1 / 2}\left(Y_{1}-k\right)=k Y_{2}^{-1 / 2}
\end{aligned}
$$

is the distance between $\mathcal{H}_{x}$ and the parallel support hyperplane of $\mathcal{K}$ lying in $\mathcal{H}_{x}^{+}$. Since either the source or the destination of each moving point must lie in $\mathcal{H}_{x}^{+} \cap \mathcal{K}$,

$$
\left\langle u, v_{i}\right\rangle \leq \min (\Theta(1), \max (\eta / t, \eta /(1-t)))=O(\min (1, \eta / t)) .
$$

Therefore

$$
\mathrm{E} \triangle\left(\mathcal{H}_{x}\right)=O(1)(V / \eta) \min (1, \eta / t) .
$$

To bound $\Gamma\left(\mathcal{H}_{x}\right)$, we call

$$
C=(\left(1-\left(y_{1} / 2\right)\right), \underbrace{0, \ldots, 0}_{d_{1}-1},\left(1-\left(y_{2} / 2\right)\right), \underbrace{0, \ldots, 0}_{d_{2}-1}, \ldots,\left(1-\left(y_{k} / 2\right)\right), \underbrace{0, \ldots, 0}_{d_{k}-1})
$$


the "center" of $\mathcal{L}$. If a moving point passes through $C$ at time $t$, its eventual destination lies inside $\mathcal{Y}=(((1-t) / t) \mathcal{K}+C) \cap \mathcal{K}$; this body is the Cartesian product of $k$ lenses and has volume $\prod_{i=1}^{k} L_{d_{i}}\left(\left(1-\left(y_{i} / 2\right)\right) / t,(1-t) / t\right)$. To each destination $Y \in \mathcal{Y}$ there corresponds a set of sources $\mathcal{X}_{Y}$ with volume $\Theta(V)$. Therefore,

$$
\Gamma\left(\mathcal{H}_{x}\right)=\Omega(\hat{\Gamma}), \quad \text { where } \quad \hat{\Gamma}=V \prod_{i=1}^{k} L_{d_{i}}\left(\left(1-\left(y_{i} / 2\right)\right) / t,(1-t) / t\right) .
$$

To bound $G\left(\mathcal{H}_{x}\right)$, we note that

$$
G\left(\mathcal{H}_{x}\right) \leq(V / \eta) \max _{p \in \mathcal{H}_{x}} f_{t}(p)
$$

It should be clear from the discussion in Section 2 that, for

$$
p=(\left(1-z_{1}\right), \underbrace{0, \ldots, 0}_{d_{1}-1},\left(1-z_{2}\right), \underbrace{0, \ldots, 0}_{d_{2}-1}, \ldots,\left(1-z_{k}\right), \underbrace{0, \ldots, 0}_{d_{k}-1}),
$$

we have

$$
f_{t}(p)=(1-t)^{-d} \prod_{i=1}^{k} L_{d_{i}}\left(\left(1-z_{i}\right) / t,(1-t) / t\right)=O(1) \cdot \prod_{i=1}^{k}\left(z_{i} / t\right)^{\left(d_{i}+1\right) / 2} .
$$

Using the method of Lagrange multipliers [15, p. 220], we maximize

$$
F\left(z_{1}, z_{2}, \ldots, z_{k}, \mu\right)=\prod_{i=1}^{k} z_{i}^{\left(d_{i}+1\right) / 2}+\mu\left(\sum_{i=1}^{k} \frac{z_{i}}{y_{i}}-k\right)
$$

to find that $f_{t}$ is maximized on $\mathcal{H}_{x}$ when

$$
z_{i}=\frac{k\left(d_{i}+1\right)}{d+k} y_{i}=\Theta\left(y_{i}\right)
$$

It follows that

$$
G\left(\mathcal{H}_{x}\right)=O(1)(V / \eta) \prod_{i=1}^{d} \min \left(1,\left(y_{i} / t\right)^{\left(d_{i}+1\right) / 2}\right) .
$$

Returning to (6.8), we note that $\exp (-n \Gamma) \leq 1, G \leq 1, \min (1, \eta / t) \leq 1$, and, since $Y_{2} \geq y_{i}^{-2}$,

$$
Y_{2}^{-(d+1) / 2} \prod_{i=1}^{k} y_{i}^{-\left(d_{i}+1\right)} \leq\left(\prod_{i=1}^{k} y_{i}^{d_{i}+(1 / k)}\right)\left(\prod_{i=1}^{k} y_{i}^{-\left(d_{i}+1\right)}\right) \leq \prod_{i=1}^{k} y_{i}^{-1+(1 / k)} .
$$

Setting $\beta=n^{-k\left(d+\left(2 /\left(d_{1}+1\right)\right)\right)}$, we see that the contribution of all cases with at least one $y_{i} \leq \beta$ is

$$
\begin{aligned}
\mathrm{E} T_{n}^{\left[\exists i: y_{i} \leq \beta\right]} & =O\left(n^{d+1}\right) \cdot k \int_{0}^{1} \cdots \int_{0}^{1} \int_{0}^{\beta} \int_{0}^{1 / 2}\left(\prod_{i=1}^{k} y_{i}^{-1+(1 / k)}\right) d t d y_{1} \cdots d y_{k} \\
& =O\left(n^{d+1}\right) \int_{0}^{\beta} y_{1}^{-1+(1 / k)} d y_{1} \\
& =O\left(n^{d+1} \beta^{1 / k}\right)=O\left(n^{\left(d_{1}-1\right) /\left(d_{i}+1\right)}\right) .
\end{aligned}
$$


If all $y_{i}>\beta$, we note that $Y_{2}^{-(d+1) / 2}=\Theta\left(\eta^{d+1}\right)$ and $\prod_{i=1}^{k} y_{i}^{-\left(d_{i}+1\right)}=V^{-2}$, giving

$$
\begin{aligned}
\mathrm{E} T_{n}-\mathrm{E} T_{n}^{\left[\exists i: y_{i} \leq \beta\right]} \\
=O\left(n^{d+1}\right) \int_{\beta}^{1} \cdots \int_{\beta}^{1} \int_{0}^{1 / 2} \exp (-n \hat{\Gamma})(\hat{\Gamma} / \eta)^{d+1}((V / \eta) \min (1, \eta / t)) \\
\quad \cdot \eta^{d+1} V^{-2} d t d y_{1} \cdots d y_{k} \\
=O(1) \int_{\beta}^{1} \cdots \int_{\beta}^{1} \int_{0}^{1 / 2} \exp (-n \hat{\Gamma})(n \hat{\Gamma})^{d+1} \min \left(\eta^{-1}, t^{-1}\right) V^{-1} d t d y_{1} \cdots d y_{k} .
\end{aligned}
$$

If $t \leq \eta$, then $\min \left(\eta^{-1}, t^{-1}\right)=\eta^{-1}$ and $\left(y_{i} / t\right)=(\eta / t)\left(r / r_{i}\right)(1 / k) \geq 1 / k=\Omega(1)$, so

$$
\hat{\Gamma}=V \prod_{i=1}^{k} L_{d_{i}}\left(\left(1-\left(y_{i} / 2\right)\right) / t,(1-t) / t\right)=\Theta(V) .
$$

So the contribution of this case is

$$
\begin{aligned}
\mathrm{E} T_{n}^{[t \leq \eta]}= & O(1) \int_{\beta}^{1} \cdots \int_{\beta}^{1} \int_{0}^{\eta} e^{-n \hat{\Gamma}}(n \hat{\Gamma})^{d+1} \eta^{-1} \hat{\Gamma}^{-1} d t d y_{1} \cdots d y_{k} \\
= & O(1) \int_{\beta}^{1} \cdots \int_{\beta}^{1} e^{-n \hat{\Gamma}}(n \hat{\Gamma})^{d+1} \hat{\Gamma}^{-1} d y_{1} \cdots d y_{k} \\
= & O(1) \int_{\beta}^{1} \cdots \int_{\beta}^{1} \int_{0}^{\infty} e^{-\tau} \tau^{d+1}\left(\frac{\tau}{n}\right)^{-1}\left(\frac{y_{1}}{\tau}\right) d \tau d y_{2} \cdots d y_{k} \\
= & O(n) \int_{\beta}^{1} \cdots \int_{\beta}^{1} \int_{0}^{\infty} e^{-\tau} \tau^{d-1}\left(\left(\frac{\tau}{n}\right)^{2 /\left(d_{1}+1\right)}\left(\prod_{i=2}^{k} y_{i}^{-\left(d_{i}+1\right) /\left(d_{1}+1\right)}\right)\right) \\
& \cdot d \tau d y_{2} \cdots d y_{k} \\
= & O\left(n^{\left(d_{1}-1\right) /\left(d_{1}+1\right)}\right)\left(\int_{0}^{\infty} e^{-\tau} \tau^{d-1+2 /\left(d_{1}+1\right)} d \tau\right)\left(\prod_{i=2}^{k} \int_{\beta}^{1} y_{i}^{-\left(d_{i}+1\right) /\left(d_{1}+1\right)} d y_{i}\right) \\
= & O\left(n^{\left(d_{1}-1\right) /\left(d_{1}+1\right)} \log ^{m-1} n\right) .
\end{aligned}
$$

If $t>\eta$, then $\min \left(\eta^{-1}, t^{-1}\right)=t^{-1}$,

$$
\hat{\Gamma}=V \prod_{i=1}^{k} \min \left(1,\left(y_{i} / t\right)^{\left(d_{i}+1\right) / 2}\right) \quad \text { and } \quad V^{-1}=\hat{\Gamma}^{-1} \prod_{i=1}^{k} \min \left(1,\left(y_{i} / t\right)^{\left(d_{i}+1\right) / 2}\right),
$$

so the contribution of this case is

$$
\begin{aligned}
\mathrm{E} T_{n}^{[t>\eta]}= & O(1) \int_{\beta}^{1} \cdots \int_{\beta}^{1} \int_{\eta}^{1 / 2} e^{-n \hat{\Gamma}}(n \hat{\Gamma})^{d+1} \hat{\Gamma}^{-1}\left(\prod_{i=1}^{k} \min \left(1,\left(y_{i} / t\right)^{\left(d_{i}+1\right) / 2}\right)\right) \\
& \cdot t^{-1} d t d y_{1} \cdots d y_{k} \\
= & O(1) \int_{\beta}^{1} \cdots \int_{\beta}^{1} e^{-n \hat{\Gamma}}(n \hat{\Gamma})^{d+1} \hat{\Gamma}^{-1}(-\log \eta) d y_{1} \cdots d y_{k} \\
= & O(\log n) \int_{\beta}^{1} \cdots \int_{\beta}^{1} e^{-n \hat{\Gamma}}(n \hat{\Gamma})^{d+1} \hat{\Gamma}^{-1} d y_{1} \cdots d y_{k} .
\end{aligned}
$$


The last equation comes from minimizing $-\log \eta$ by simultaneously minimizing the $y_{i}$ at $\beta$. The remaining integral is identical to (6.12). Therefore, we have as our final result

$$
\mathrm{E} T_{n}=O\left(n^{\left(d_{1}-1\right) /\left(d_{1}+1\right)} \log ^{m} n\right)
$$

\section{Convex Hull Transitions under Normal Distribution}

The $d$-dimensional standard normal (or Gaussian) distribution lends itself to easy analysis, since it is both a spherically symmetric distribution and a product distribution-the $d$ coordinates of a point are themselves independent and normally distributed. For moving points, we have

$$
\begin{aligned}
\mathrm{E} T_{n} & =\Theta\left(n^{d+1}\right) \int \exp (-n \Gamma(c, t)) G(c, t)^{d+1} \mathrm{E} \triangle(c, t) r^{-(d-1)} d \lambda(c) d t \\
& =\Theta\left(n^{d+1}\right) \int \exp (-n \Gamma(c, t)) G(c, t)^{d+1} \mathrm{E} \triangle(c, t) d t d r d \sigma(u) \\
& =\Theta\left(n^{d+1}\right) \int_{0}^{\infty} \int_{0}^{1} \exp (-n \Gamma(r, t)) G(r, t)^{d+1} \mathrm{E} \triangle(r, t) d t d r
\end{aligned}
$$

We note that $G(r, t)$ is just the density of the probability that

$$
P_{i}(t)=(1-t) X_{i}^{(1)}+t Y_{i}(1)=r
$$

and, according to somewhat lengthy but routine computations,

$$
\begin{aligned}
G(r, t) & =\int_{-\infty}^{\infty}\left(\frac{1}{(1-t) \sqrt{2 \pi}} \exp \left(-\frac{1}{2}\left(\frac{r-x}{1-t}\right)^{2}\right)\right)\left(\frac{1}{t \sqrt{2 \pi}} \exp \left(-\frac{1}{2}\left(\frac{x}{t}\right)^{2}\right)\right) d x \\
& =\frac{1}{\sqrt{2 T \pi}} \exp \left(-\frac{r^{2}}{2 T}\right),
\end{aligned}
$$

where

$$
T=t^{2}+(1-t)^{2}=\Theta(1)
$$

Likewise,

$$
\Gamma(r, t)=\int_{r}^{\infty} G(x, t) d x \sim(T / r) G(r, t) .
$$

To estimate $\mathrm{E} \triangle(r, t)$, we let $\mathrm{E} \triangle^{\prime}(r, t)$, represent the $(d-1)$-volume of the convex hull of the $d+1$ points on hyperplane $\mathcal{H}$. Because the normal density is a product density, $\mathrm{E} \triangle^{\prime}(r, t)$ is independent of both $r$ and $\left\langle u, v_{i}\right\rangle$ for $1 \leq i \leq d+1$. 
Therefore, we have

$$
\mathrm{E} \triangle(r, t) \leq \mathrm{E}\left(\triangle^{\prime}(r, t)\right) \cdot \mathrm{E}\left(\max _{i: 1 \leq i \leq d+1}\left\langle u, v_{i}\right\rangle \mid(1-t) X_{i}^{(1)}+t Y_{i}^{(1)}=r\right) .
$$

Now

$$
\begin{aligned}
& \mathrm{E}\left(\max _{i: 1 \leq i \leq d+1}\left\langle u, v_{i}\right\rangle \mid(1-t) X_{i}^{(1)}+t Y_{i}^{(1)}=r\right) \\
& \leq \mathrm{E}\left(\sum_{i: 1 \leq i \leq d+1}\left\langle u, v_{i}\right\rangle \mid(1-t) X_{i}^{(1)}+t Y_{i}^{(1)}=r\right) \\
& \leq(d+1) \mathrm{E}\left(\left\langle u, v_{1}\right\rangle \mid(1-t) X_{1}^{(1)}+t Y_{1}^{(1)}=r\right) \\
& \leq \Theta(1) \mathrm{E}\left(\frac{\left|r-X_{1}^{(1)}\right|}{t} \mid(1-t) X_{1}^{(1)}+t Y_{1}^{(1)}=r\right) \\
& \leq \frac{\Theta(1)}{G(r, t)} \int_{-\infty}^{\infty} \frac{|r-x|}{t}\left(\frac{1}{(1-t) \sqrt{2 \pi}} \exp \left(-\frac{1}{2}\left(\frac{x}{1-t}\right)^{2}\right)\right) \\
& \times\left(\frac{1}{t \sqrt{2 \pi}} \exp \left(-\frac{1}{2}\left(\frac{r-x}{t}\right)^{2}\right)\right) d x \\
&= \Theta\left(\frac{r t^{2}}{2 \pi T}\right),
\end{aligned}
$$

and, similarly,

$$
\begin{aligned}
\mathrm{E}\left(\triangle^{\prime}(t)\right) & \leq \Theta(1) \cdot(d+1) \mathrm{E}\left(\left|(1-t) X_{1}+t Y_{1}-(r, 0, \ldots, 0)\right|^{d-1} \mid(1-t) X_{1}^{(1)}+t Y_{1}^{(1)}=r\right) \\
& \leq \Theta(1) \cdot(d+1)(d-1) \mathrm{E}\left(\left|(1-t) X_{1}^{(2)}+t Y_{1}^{(2)}\right|^{d-1}\right) \\
& =\Theta(1) \int_{-\infty}^{\infty} \int_{-\infty}^{\infty}|(1-t) x+t y|^{d-1}\left(\frac{e^{-x^{2} / 2}}{\sqrt{2 \pi}}\right)\left(\frac{e^{-y^{2} / 2}}{\sqrt{2 \pi}}\right) d x d y \\
& =\Theta\left(T^{(d-1) / 2}\right) .
\end{aligned}
$$

So $\mathrm{E} \triangle(r, t)=\Theta\left(t^{2} r\right)$.

Finally, setting $\alpha=n \Gamma(r, t), d \alpha=n d \Gamma=n G d r$, and $r \sim \sqrt{2 \log (n / \alpha)}$ in (7.1), we obtain, as desired,

$$
\begin{aligned}
\mathrm{E} T_{n} & =\Theta\left(n^{d+1}\right) \int_{0}^{1} \int_{0}^{n / 2} e^{-\alpha}(\alpha r / n)^{d} r t^{2}(d \alpha / n) d t \\
& =\Theta(1) \int_{0}^{n / 2} e^{-\alpha} \alpha^{d} r^{d+1} d \alpha \\
& =\Theta(1) \int_{0}^{n / 2} e^{-\alpha} \alpha^{d}(2 \log (n / \alpha))^{(d+1) / 2} d \alpha \\
& =\Theta\left(\log ^{(d+1) / 2} n\right) .
\end{aligned}
$$




\section{References}

1. F. Affentranger and J. A. Wieacker, On the convex hull of uniform random points in a simple $d$-polytope. Discrete \& Computational Geometry 6 (1991), 291-305.

2. P. K. Agarwal, L. Guibas, J. Hershberger, and E. Veach, Maintaining the extent of a moving point set, in Proc. 5th Workshop on Algorithms and Data Structures, pp. 31-44. Lecture Notes in Computer Science, Vol. 1272. Springer-Verlag, Berlin, 1997.

3. G. Albers and T. Roos, Voronoi diagrams of moving points in higher dimensional spaces, in Proc. 3 rd Scandinavian Workshop on Algorithm Theory, pp. 399-409, 1992.

4. J. Basch, H. Devarajan, P. Indyk, and L. Zhang, Probabilistic analysis for combinatorial functions of moving points. Manuscript, 1998.

5. R. A. Dwyer, Random convex hulls in a product of balls. Probability Theory and Related Fields $\mathbf{8 6}$ (1990), 457-467.

6. R. A. Dwyer, Higher-dimensional Voronoi diagrams in linear expected time. Discrete \& Computational Geometry 6 (1991), 343-367.

7. R. A. Dwyer, Maximal and minimal balls. Computational Geometry: Theory and Applications 3 (1993), 261-275.

8. R. A. Dwyer, Voronoi diagrams of random lines and flats. Discrete \& Computational Geometry 17 (1997), 123-136.

9. L. Guibas, J. S. B. Mitchell, and T. Roos, Voronoi diagrams of moving points in the plane, in Proc. 17th Internat. Workshop on Graph-Theoretical Concepts in Computer Science, pp. 113-125. Lecture Notes in Computer Science, Vol. 570. Springer-Verlag, Berlin, 1991.

10. H. Raynaud, Sur l'envelope convexe des nuages des points aléatoires dans $\mathbf{R}^{n}$, I. Journal of Applied Probability 7 (1970), 35-48.

11. A. Rényi and R. Sulanke, Über die konvexe Hülle von $n$ zufällig gewählten Punkten. Zeitschrift für Wahrscheinlichkeitstheorie und Verwardte Gebiete 2 (1963), 75-84 and 3 (1964), 138-147.

12. T. Roos, Voronoi diagrams over dynamic scenes. Discrete Applied Mathematics, 43 (1993), 243-259.

13. L. Santaló, Integral Geometry and Geometric Probability. Encyclopedia of Mathematics and Its Applications, Vol. 1. Addison-Wesley, Reading, MA, 1976.

14. M. I. Shamos, Computational Geometry. Ph.D. thesis, Yale University, New Haven, CT, 1978.

15. H. S. Wilf, Mathematics for the Physical Sciences, Dover, New York, 1962.

Received November 23, 1998, and in revised form July 8, 1999. 